\title{
Impact of Foreign Official Purchases of U.S. Treasuries on the Yield Curve
}

\author{
Erin L. Wolcott \\ Online Appendix
}

As in Hamilton and $\mathrm{Wu}$ (2012), the mapping between reduced-form and structural parameters follows:

$$
\begin{aligned}
& \phi_{m m}^{*}=\left[\rho_{1} \rho_{2} \ldots \rho_{12}\right] \\
& A_{1}^{*}=A_{1}-B_{1 \ell} \rho_{\ell \ell} B_{1 \ell}^{-1} A_{1} \\
& \phi_{1 m}^{*}=\left[\begin{array}{rr}
B_{1 m}^{(1)} & 0
\end{array}\right]-B_{1 \ell} \rho_{\ell \ell} B_{1 \ell}^{-1}\left[\begin{array}{cc}
B_{1 m}^{(0)} & B_{1 m}^{(1)}
\end{array}\right] \\
& \phi_{11}^{*}=B_{1 \ell} \rho_{\ell \ell} B_{1 \ell}^{-1} \\
& \psi_{1 m}^{*}=B_{1 m}^{(0)} \\
& A_{2}^{*}=A_{2}-B_{2 \ell} B_{1 \ell}^{-1} A_{1} \\
& \phi_{2 m}^{*}=B_{2 m}-B_{2 \ell} B_{1 \ell}^{-1} B_{1 m} \\
& \phi_{21}^{*}=B_{2 \ell} B_{1 \ell}^{-1} \\
& \operatorname{Var}\left[\begin{array}{c}
u_{m t}^{*} \\
u_{1 t}^{*} \\
u_{2 t}^{*}
\end{array}\right]=\left[\begin{array}{ccc}
\Omega_{m}^{*} & 0 & 0 \\
0 & \Omega_{1}^{*} & 0 \\
0 & 0 & \Omega_{2}^{*}
\end{array}\right]=\left[\begin{array}{ccc}
\Sigma_{m m} \Sigma_{m m}^{\prime} & 0 & 0 \\
0 & B_{1 \ell} B_{1 \ell}^{\prime} & 0 \\
0 & 0 & \Sigma_{e} \Sigma_{e}^{\prime}
\end{array}\right],
\end{aligned}
$$

where $\hat{\Sigma}_{m m}$ is the Cholesky factorization of $\hat{\Omega}_{m}^{*}$ and $\hat{\Sigma}_{e}$ is the square root of the diagonal elements of $\hat{\Omega}_{2}^{*} \cdot{ }^{1}$ Additionally, $A_{1}, A_{2}, B_{1}, B_{2}$ are defined as:

$$
\begin{gathered}
{\left[\begin{array}{l}
A_{1} \\
A_{2}
\end{array}\right]=\left[\begin{array}{l}
\alpha_{12} \\
\alpha_{36} \\
\alpha_{72} \\
\alpha_{24} \\
\alpha_{48} \\
\alpha_{60}
\end{array}\right],} \\
{\left[\begin{array}{lll}
B_{1 m}^{(0)} & B_{1 m}^{(1)} & B_{1 \ell} \\
B_{2 m}^{(0)} & B_{2 m}^{(1)} & B_{2 \ell}
\end{array}\right]=\left[\begin{array}{c}
\beta_{12}^{\prime} \\
\beta_{36}^{\prime} \\
\beta_{72}^{\prime} \\
\beta_{24}^{\prime} \\
\beta_{48}^{\prime} \\
\beta_{60}^{\prime}
\end{array}\right],}
\end{gathered}
$$

where for $i=1,2, B_{i m}^{(0)}$ are $(3 \times 4)$ matrices relating the observed yields to the 4 contemporaneous macro factors. $B_{i m}^{(1)}$ are $(3 \times 44)$ matrices relating the observed yields to 11 lags of the 4 macro factors. Lastly, $B_{i \ell}$ are $(3 \times 3)$ matrices relating the observed yields to the latent factors.

\footnotetext{
${ }^{1}$ Macro variables in $f_{t}^{m}$ are ordered as follows: output growth, inflation, dollar appreciation, foreign official purchases scaled by publicly held Treasury notes and bonds outstanding.
} 
Figure 1: Foreign Official Holdings of US Treasuries

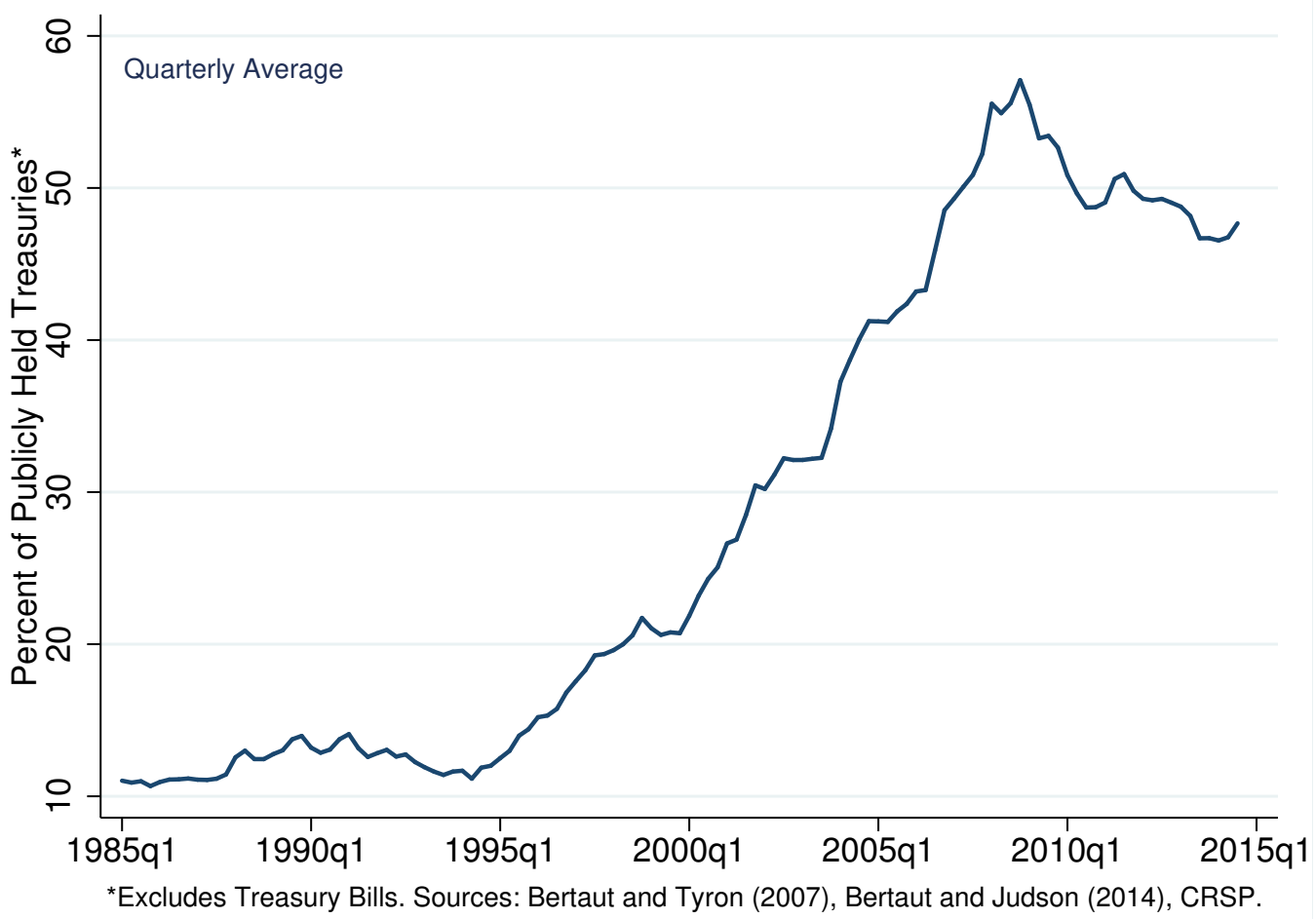

Figure 2: Maturity Structure of Foreign Official Holdings of US Treasuries

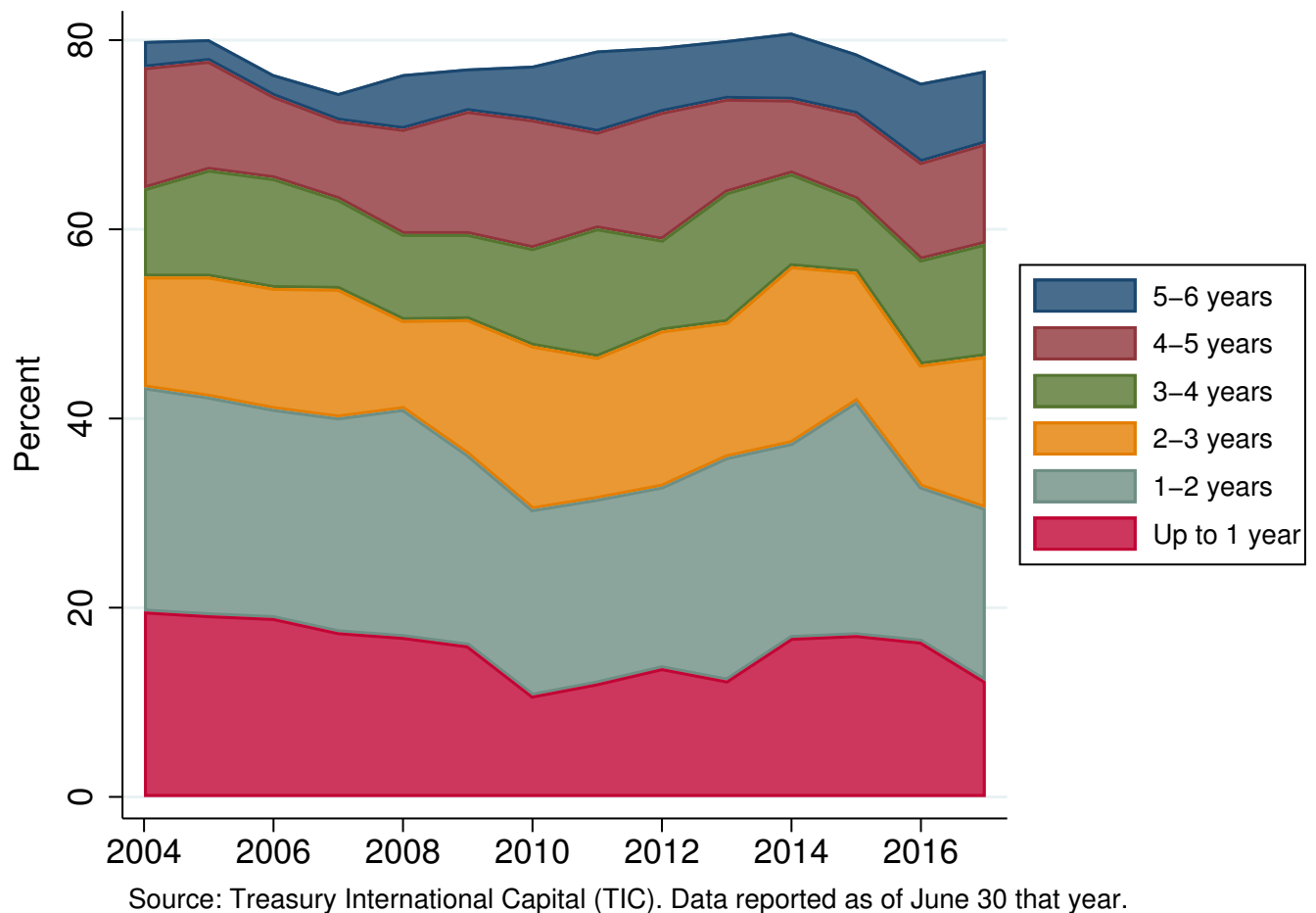


Figure 3: Scaling Net Foreign Official Purchases by Treasuries Outstanding
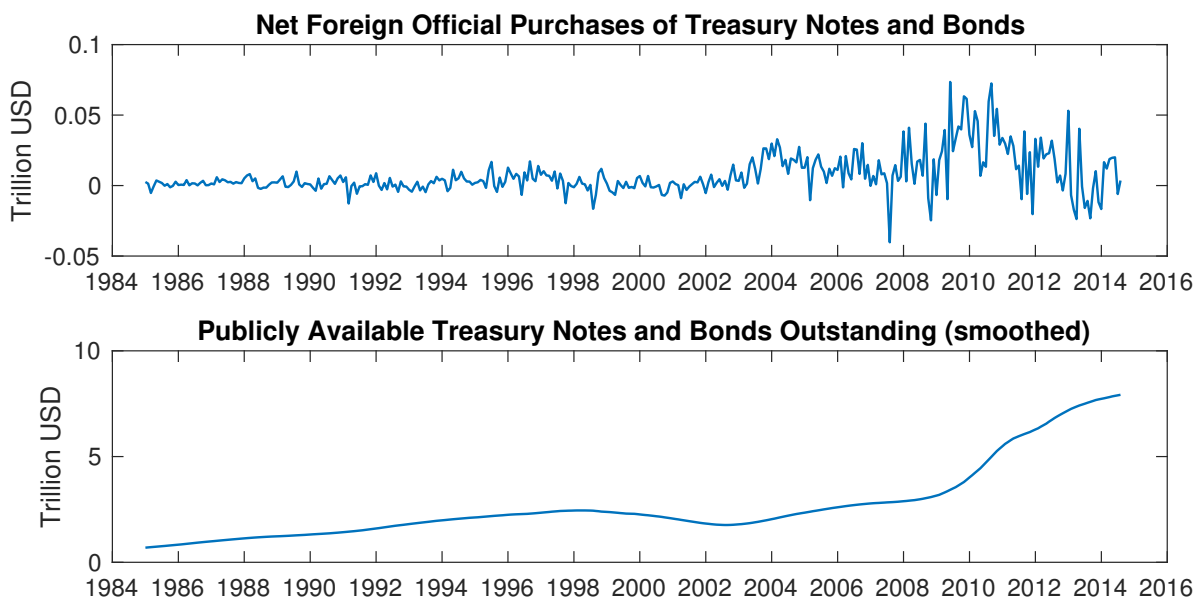

Ratio

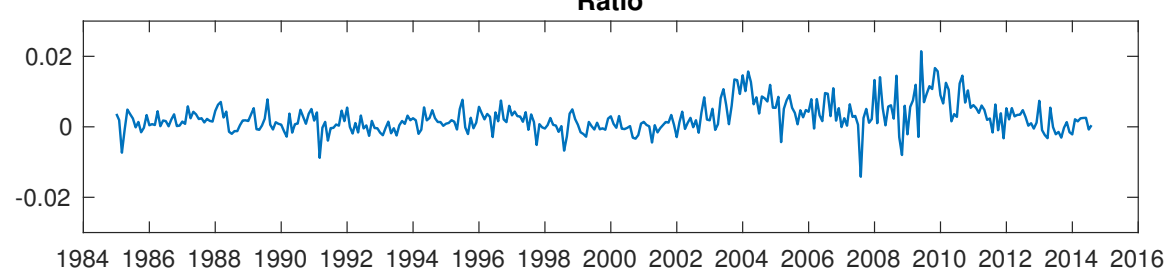

Figure 4: Response of Macro Variable SVAR to Foreign Official Purchase Shock*
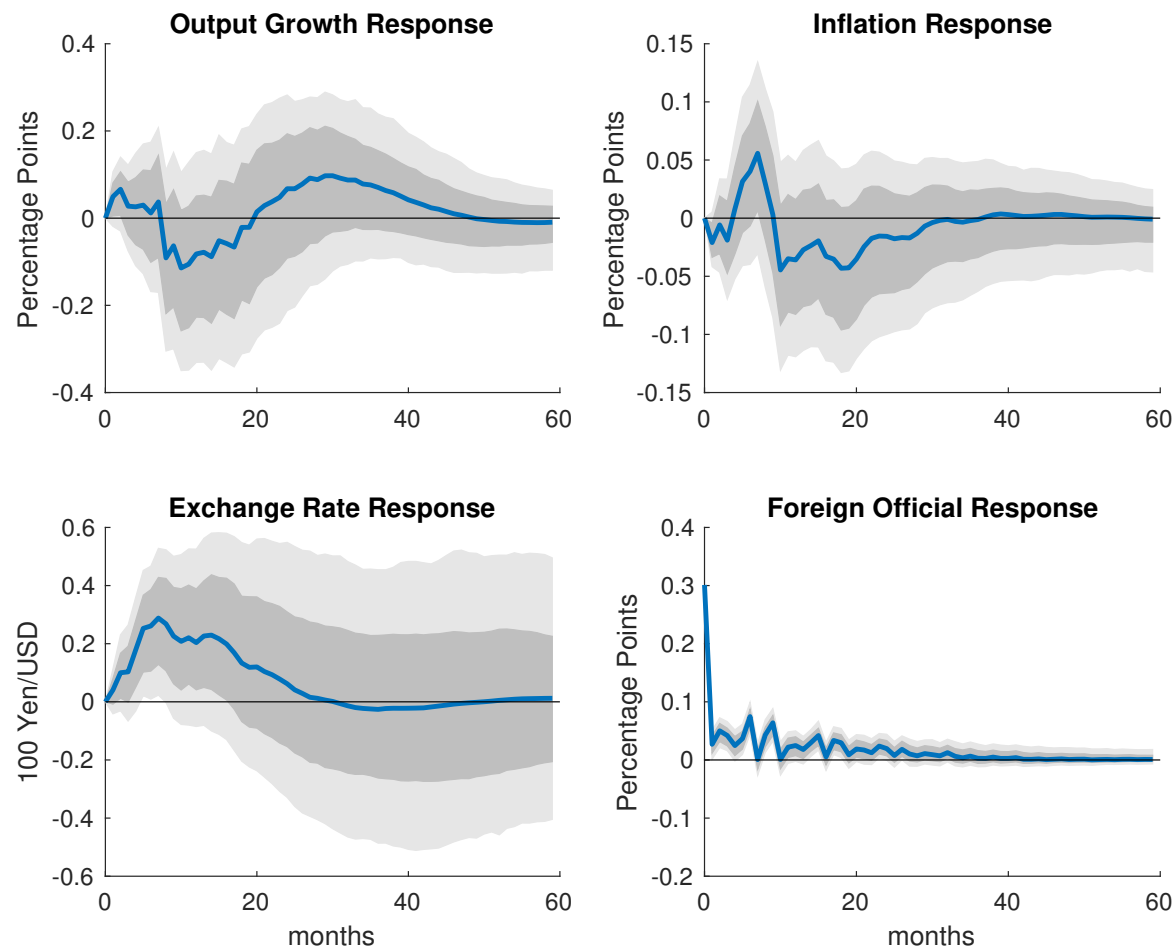

*68\% and $90 \%$ bootstrapped confidence intervals plotted 
Figure 5: Impulse Response Functions with Total Foreign Purchases
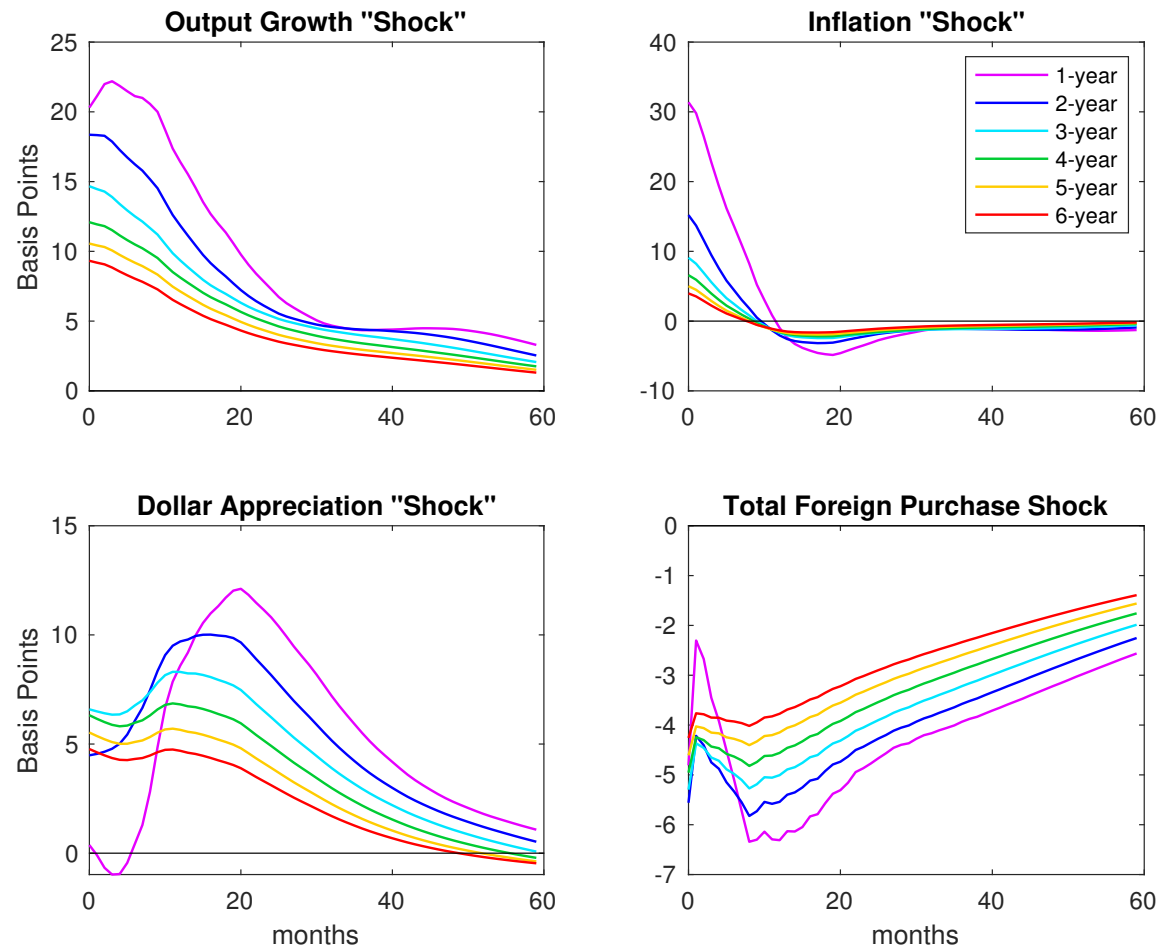

Figure 6: Impulse Response Functions Excluding the Great Recession*
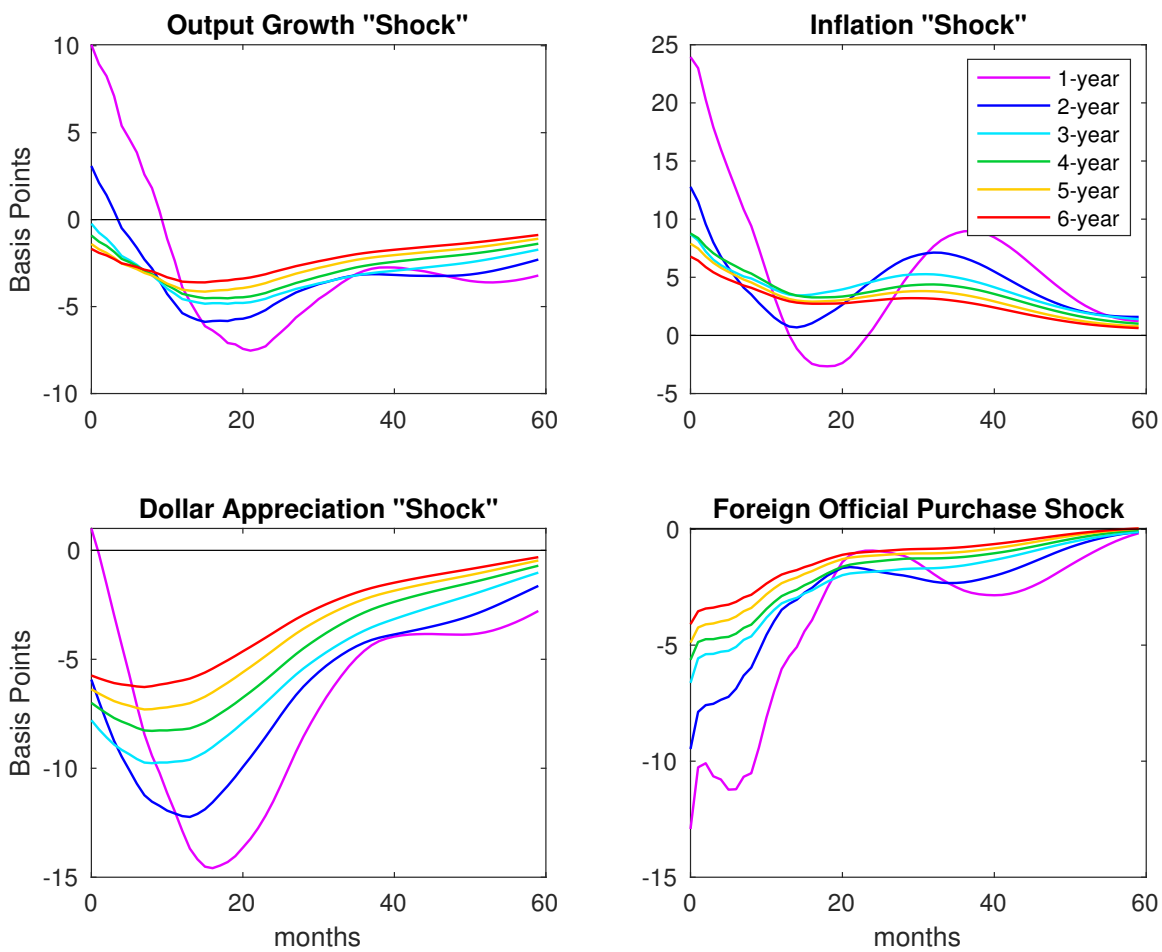

*Sample period 1985m1-2007m11 